be one of the highest predicted for a photochemical reaction not involving a chain sequence.

Margaret I. Christie Bruce M. Coluins

Inorganic Chemistry Laboratory,

University of Oxford.

Received May 8, 1968.

${ }^{1}$ Cvetanovic, R. J., Canad. J. Chem., 34, 775 (1956).

${ }^{2}$ Avery, H. E., and Cvetanovié, R. J., J. Chem. Phys., 43, 3727 (1965).

${ }^{3}$ Avramenko, L. I., and Kolesnikova, R. V., Adv. Photochem., 2, 25 (1964).

'Leighton, P. A., Photochemistry of Air Pollution, 55 (Academic Press,

${ }^{8}$ Phillips, L., and Shaw, R., Tenth Combustion Symp., 453 (1965).

- Cvetanovic, R. J., Adv. Photochem., 1, 115 (1963).

${ }^{7}$ Klein, F. S., and Herron, J. T., J. Chem. Phys., 41, 1285 (1964).

'Cadle, R. D., and Powers, J. W., J. Phys. Chem., 71, 1702 (1967).

\section{Habit Changes in Mixed Crystals of Phenazine and $\mathbf{N}$-Oxyphenazine}

WE recently measured and plotted against composition ${ }^{1}$ the refractive indices of a complete mixed crystal series of phenazine and $N$-oxyphenazine, crystallized from methyl ethyl ketone. We originally thought that the crystals, which were tabular, showed the same habit, the best-developed faces being (201), but we have since found this to be a mistake. A more careful study of the morphology, using X-ray oscillation photographs, has shown that the \{hol\} habit does actually change across the series (Fig. 1). Although the relative sizes of the crystal faces vary in the series, the tabular appearance is obtained at all concentrations, except in a region of poor development.

Crystals of pure phenazine ( 0 per cent $N$-oxyphenazine) nearly always have large (100) faces, though occasionally some with large (201) faces are found. Crystals containing about 28 mole per cent $N$-oxyphenazine are badly de. veloped, and tend to be fibrous. X-ray photographs of two of these crystals that were sufficiently well developed showed that in one case the largest face was (201), in the other it was (001). This may indicate that there is a eutectic at or near this composition.

These results make it clear that the refractive index versus composition curve previously given for measurements made perpendicular to the $b$ axis, is not a real measure of that quantity. Instead, it reflects the change in morphology as the composition varies. From the large apparent peak at about 8 mole per cent $N$-oxyphenazine, it is evident that only a small amount of $N$-oxyphenazine is sufficient to bring about a considerable change in the habit of the phenazine crystals. In this case, the crystal habit is a function of composition, as has been found with some other mixed crystal systems ${ }^{2-4}$, although in the solid-solution series of tetraphenylporphin and silver tetraphenylporphin the crystal habit was not found to be a function of composition ${ }^{5}$.

The measurements made parallel to the $b$ axis do, however, refer to genuine refractive index changes, with

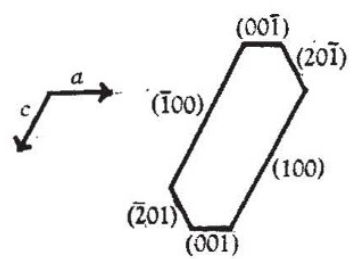

$\boldsymbol{a}$

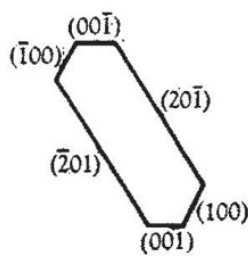

$b$

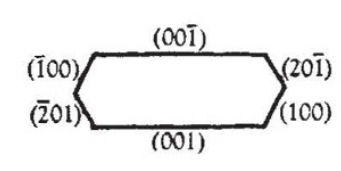

$c$
Fig. 1. \{hol\} Habit changes of mixed crystals of phenazine and $N$-oxyphenazine. Compositions are given in terms of mole per cent $N$-oxyphenazine. $a, 0$ per cent; $b$, occasionally per cent. respect to the particular erystal direction [010], and show a discontinuity between about 8 and 30 mole per cent $N$-oxyphenazine. This lends support to the view that there is a eutectic region in this mixed crystal system.

Some crystals in this region of composition were examined microscopically at low temperature (about $80^{\circ} \mathrm{K}$ ), but no apparent onset of inhomogeneity was observed. It would be interesting to see whether the change in morphology across the series is independent of the solvent used.

A. M. Glazer

S. Pramatus

Department of Chemistry,

University College,

University of London.

Received May 13, 1968.

1 Glazer, A. M., and Pramatus, 8., Nature, 217, 58 (1968).

2 Kitaigorodskii, A. I., and Myasnikova, R. M., Kristallografiya, 3, 160 (1958).

${ }^{3}$ Kolosov, N. Ia., Kristallografiya, 3, 700 (1958).

“ Kitaigorodskii, A. I., Myasnikova, R. M., and Samarskaya, v. D., Kristallografiya, 8, 393 (1983).

'Donnay, G., and Storm, C. B., Molecular Crystals, 2, 287 (1967).

\section{Correlation between the Nitrogen Impurity Content and the Crystal Habit of Synthetic Diamond}

THE presence of nitrogen in diamond has provided an explanation for some of the variations which are observed in the physical properties of this material ${ }^{1-3}$. The nitrogen impurity can exist either as dispersed substitutional donors, in which case electron spin resonance is observed ${ }^{2}$, or as non-paramagnetic aggregates of atoms ${ }^{3}$. Absorption peaks in the infrared can be obtained from nitrogen in either form ${ }^{4}$. Peaks at $1,129 \mathrm{~cm}^{-1}$ and $1,345 \mathrm{~cm}^{-1}$, coupled by a broad saddle which is peaked at $1,290 \mathrm{~cm}^{-1}$, are characteristic of the paramagnetic centres. In contrast, aggregates of nitrogen give a single peak at $1,280 \mathrm{~cm}^{-1}$. Dyer et al..$^{4}$ have shown that the two forms of nitrogen can be distinguished even if they co-exist in a particular specimen. In most cases, however, the nitrogen exists predominantly in one form and the diamond is classified either as type $1 a$ or as type $1 b$. In type la diamond, the aggregates of nitrogen are in a majority and in type $1 b$ diamond the concentration of dispersed nitrogen is the larger.

In a recent survey of $M B S 35 / 40$ synthetic diamond (mean size 480u) (manufactured by the General Electric Company) by electron spin resonance, $w^{5}$ found that, unlike most stones which exhibited the electron resonance associated with dispersed substitutional nitrogen (type 1b), a small number of stones gave no detectable resonance. Diamonds of this type were invariably almost perfect octahedra whereas the usual habit for $M B S 35 / 40$ diamond is cubo-octahedral. The octahedra have now been examined both by electron spin resonance and by infrared spectroscopy.

Of the small number of octahedra which were available, only two were sufficiently free from inclusions to be investigated. The stones gave no observable electron resonance signal characteristic of dispersed nitrogen, while the infrared peaks associated with either the dispersed or aggregated nitrogen were scarcely detectable.

Cubo-octahedral diamonds which had previously been examined by electron spin resonance and were known to contain approximately $10^{17}$ nitrogen donors/ $\mathrm{cm}^{3}$ gave infrared peaks at $1,129 \mathrm{~cm}^{-1}$ and $1,345 \mathrm{~cm}^{-1}$, the amplitudes of which were several times greater than the noise level. 